\title{
Lyman- $\alpha$ Forest Constraints on Primordial Black Holes as Dark Matter
}

\author{
Riccardo Murgia, ${ }^{1,2,3}$ Giulio Scelfo, ${ }^{1,2,3}$ Matteo Viel, ${ }^{1,2,3,4}$ and Alvise Raccanelli ${ }^{5}$ \\ ${ }^{1}$ SISSA, Via Bonomea 265, 34136 Trieste, Italy \\ ${ }^{2}$ INFN, Sez. di Trieste, via Valerio 2, 34127 Trieste, Italy \\ ${ }^{3}$ IFPU, Institute for Fundamental Physics of the Universe, via Beirut 2, 34151 Trieste, Italy \\ ${ }^{4}$ INAF/OATS, Osservatorio Astronomico di Trieste, via Tiepolo 11, I-34143 Trieste, Italy \\ ${ }^{5}$ Theoretical Physics Department, CERN, 1 Esplanade des Particules, CH-1211 Geneva 23, Switzerland
}

(Received 1 April 2019; revised manuscript received 6 June 2019; published 16 August 2019)

\begin{abstract}
The renewed interest in the possibility that primordial black holes (PBHs) may constitute a significant part of dark matter has provided motivation for revisiting old observational constraints, as well as developing new ones. We present new limits on the PBH abundance, from a comprehensive analysis of high-resolution highredshift Lyman- $\alpha$ forest data. Poisson fluctuations in the PBH number density induce a small-scale power enhancement which departs from the standard cold dark matter prediction. Using a grid of hydrodynamic simulations exploring different values of astrophysical parameters, we obtain a marginalized upper limit on the PBH mass of $f_{\mathrm{PBH}} M_{\mathrm{PBH}} \sim 60 M_{\odot}$ at $2 \sigma$, when a Gaussian prior on the reionization redshift is imposed, preventing its posterior distribution from peaking on very high values, which are disfavored by the most recent estimates obtained both through cosmic microwave background and intergalactic medium observations. Such a bound weakens to $f_{\mathrm{PBH}} M_{\mathrm{PBH}} \sim 170 M_{\odot}$ when a conservative flat prior is instead assumed. Both limits significantly improve on previous constraints from the same physical observable. We also extend our predictions to nonmonochromatic $\mathrm{PBH}$ mass distributions, ruling out large regions of the parameter space for some of the most viable PBH extended mass functions.
\end{abstract}

DOI: 10.1103/PhysRevLett.123.071102

Introduction.-Primordial black holes (PBHs) were first theorized decades ago [1]. Many proposals were made for their formation mechanism, such as collapsing large fluctuations produced during inflation [2-4], collapsing cosmic string loops [5-7], domain walls [8,9], bubble collisions [10,11], and a collapse of exotic dark matter (DM) clumps [12].

After the first gravitational-wave $(\mathrm{GW})$ detection revealed merging black hole $(\mathrm{BH})$ binaries of masses $O\left(10 M_{\odot}\right)$ $[13,14]$, the interest in PBHs as DM candidates has been revived [15]. Several proposals to determine the nature of the merging $\mathrm{BH}$ progenitors have been made, involving methods as GW-large-scale-structure cross-correlations [16,17], BH binary eccentricities [18], BH mass function studies [19,20], and lensing of fast radio bursts [21].

Several constraints on the PBH abundance have been determined through different observables, such as gravitational lensing [22-31] and dynamical [32-39] and accretion effects [40-44]. Nevertheless, varying the numerous assumptions involved might significantly alter these limits

Published by the American Physical Society under the terms of the Creative Commons Attribution 4.0 International license. Further distribution of this work must maintain attribution to the author(s) and the published article's title, journal citation, and DOI.
[45-47], making the investigation of PBHs as DM candidates still fully open. Specifically, two mass regimes are currently of large interest: $O\left(10^{-10} M_{\odot}\right)$ and $O\left(10 M_{\odot}\right)$ (see Refs. [48-50] for details).

A mostly unexplored method for constraining the PBH abundance is offered by the Lyman- $\alpha$ forest, which is the main manifestation of the intergalactic medium (IGM), and it represents a powerful tool for tracing the DM distribution at (sub)galactic scales (see, e.g., Refs. [51-53]). Lyman- $\alpha$ data were used about 15 years ago to set an upper limit of a few $10^{4} M_{\odot}$ on PBH masses, assuming all DM to be made by PBHs with the same mass [54]. In this Letter, we update and improve upon such a limit, using the highest resolution, most up-to-date Lyman- $\alpha$ forest data [53], and a new set of highresolution hydrodynamic simulations. Furthermore, we generalize our results to different $\mathrm{PBH}$ abundances and nonmonochromatic mass distributions.

Poisson noise and impact on the matter power spectrum.Stellar-mass PBHs would cause observable effects on the matter power spectrum; due to discreetness, a small-scale plateau in the linear power spectrum is induced by a Poisson noise contribution [49,54-56].

If PBHs are characterized by a monochromatic mass distribution (MMD), they are parametrized by their mass, $M_{\mathrm{PBH}}$, and abundance so that the fraction parameter $f_{\mathrm{PBH}} \equiv \Omega_{\mathrm{PBH}} / \Omega_{\mathrm{DM}}=1$ where all DM is made of PBHs. 
If PBHs are randomly distributed, their number follows a Poisson distribution, and each wave number $k$ is associated with an overdensity $\delta_{\mathrm{PBH}}(k)$ due to Poisson noise. The $\mathrm{PBH}$ contribution to the power spectrum is thus defined as

$$
P_{\mathrm{PBH}}(k)=\left\langle\left|\delta_{\mathrm{PBH}}(k)\right|^{2}\right\rangle=\frac{1}{n_{\mathrm{PBH}}},
$$

where $n_{\mathrm{PBH}}$ is the comoving $\mathrm{PBH}$ number density. Since $n_{\mathrm{PBH}}$ is a $k$-independent quantity, $P_{\mathrm{PBH}}$ is scale invariant.

One can interpret the $\mathrm{PBH}$ overdensity as an isocurvature perturbation $[54,56]$. Hence, the total power spectrum can be written as

$$
P_{\mathrm{CDM}}(k, z)=D^{2}(z)\left[T_{\mathrm{ad}}^{2}(k) P_{\mathrm{ad}}+T_{\text {iso }}^{2}(k) P_{\text {iso }}\right],
$$

where $D(z)$ is the growth factor, $P_{\text {iso }}$ is the isocurvature power spectrum, and $P_{\text {ad }} \propto A_{s} k^{n_{s}}$ is the primordial adiabatic power spectrum. $T_{\text {ad }}$ and $T_{\text {iso }}$ are the adiabatic and isocurvature transfer functions, respectively. The $\mathrm{PBH}$ linear power spectrum is thus defined by

$$
P_{\text {iso }}=f_{\mathrm{PBH}}^{2} P_{\mathrm{PBH}}=\frac{2 \pi^{2}}{k^{3}} A_{\text {iso }}\left(\frac{k}{k_{*}}\right)^{n_{\text {iso }}-1},
$$

where we set the pivot scale $k_{*}=0.05 / \mathrm{Mpc}$, and the primordial isocurvature tilt $n_{\text {iso }}=4$ in order to ensure the scale invariance. Since the adiabatic power spectrum evolves as $k^{-3}$ at large $k$, the isocurvature contribution is expected to become important only at the scales probed by the Lyman- $\alpha$ forest; $A_{\text {iso }}$ sets the amplitude of the isocurvature modes, depending on the $\mathrm{PBH}$ mass considered; we can then express the isocurvature-to-adiabatic amplitude ratio:

$f_{\text {iso }}=\sqrt{\frac{A_{\text {iso }}}{A_{s}}}=\sqrt{\frac{k_{*}^{3} f_{\mathrm{PBH}}^{2}}{2 \pi^{2} n_{\mathrm{PBH}}} \frac{1}{A_{\mathrm{s}}}}=\sqrt{\frac{k_{*}^{3} M_{\mathrm{PBH}} f_{\mathrm{PBH}}}{2 \pi^{2} \Omega_{\mathrm{CDM}} \rho_{\mathrm{cr}}} \frac{1}{A_{\mathrm{s}}}}$,

where the last equality holds only for MMDs. Different combinations of $\mathrm{PBH}$ mass and abundance correspond to the same isocurvature-to-adiabatic amplitude ratio if the quantity $f_{\mathrm{PBH}} M_{\mathrm{PBH}}$ is the same. In our framework, the effect on the linear matter power spectrum due to the presence of isocurvature modes consists of a power enhancement with respect to the standard lambda cold dark matter $(\Lambda \mathrm{CDM})$ spectrum, in the form of a small-scale plateau.

The Lyman- $\alpha$ forest observable is the 1D flux power spectrum, which, being a projection of 3D nonlinear matter power spectrum, is an ideal tracer for the small-scale DM distribution along our lines of sight. In Fig. 1, we show the 1D flux power for the $\Lambda$ CDM model, together with the spectra corresponding to different values of $M_{\mathrm{PBH}}$. Symbols refer to MIKE and HIRES spectrograph data. To exhibit the variations in the flux power induced by different IGM

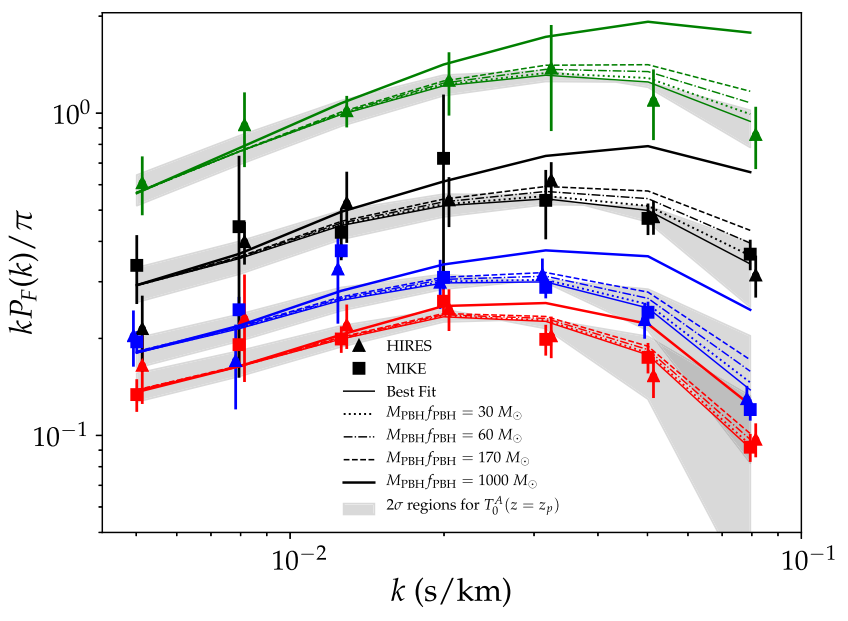

FIG. 1. 1D flux spectra for $\Lambda \mathrm{CDM}$ and $\Lambda \mathrm{PBH}$, for different PBH masses. Symbols are data from MIKE and HIRES spectrographs, lines are obtained by interpolating in the $\left(M_{\mathrm{PBH}} f_{\mathrm{PBH}}\right)$ space defined by our simulations; while the best fit is technically for $M_{\mathrm{PBH}} \neq 0$, it is indistinguishable from the $\Lambda$ CDM case. Red, blue, black, and green indicate $z=4.2,4.6,5.0,5.4$, respectively. The gray dashed areas represent regions sampled by flux power spectra corresponding to values for a $T_{0}^{A}$ spanning its marginalized $2 \sigma$ interval.

thermal histories, we also show, as gray dashed areas, the impact of different IGM temperature evolutions.

Extended mass distributions.-The PBH formation is, in the most standard case, due to large perturbations in the primordial power spectrum; while the exact details of the peak required to form a $\mathrm{PBH}$ and how this is linked to the real-space overdensities are still unclear [57], PBHs could have an extended mass function. Moreover, a nonmonochromatic mass distribution would be created by different merger and accretion history for each PBH. General methods to convert MMD constraints to limits on extended mass distributions (EMDs) were developed in Refs. [46,58].

The extension to EMDs of the observable considered here arises naturally from the second equality in Eq. (4) by directly taking the $\mathrm{PBH}$ number density corresponding to a given EMD. Consider EMDs in the form

$$
\frac{d n_{\mathrm{PBH}}}{d \ln M_{\mathrm{PBH}}}=f_{\mathrm{PBH}} \rho_{\mathrm{DM}} \frac{d \Phi_{\mathrm{PBH}}}{d M_{\mathrm{PBH}}},
$$

where the function $d \Phi_{\mathrm{PBH}} / d M_{\mathrm{PBH}}$ describes the EMD shape, and $\rho_{\mathrm{DM}}=\Omega_{\mathrm{DM}} \rho_{\mathrm{cr}}$. Given an EMD, one can define the so-called equivalent mass $M_{\text {eq }}$, which is the mass of a MMD providing the same observational effect. The conversion is given by

$$
\begin{aligned}
f_{\mathrm{PBH}}^{2} & {\left[\frac{\Omega_{\mathrm{DM}} \rho_{\mathrm{cr}} f_{\mathrm{PBH}}}{M_{\mathrm{eq}}}\right]^{-1} } \\
& =\frac{f_{\mathrm{PBH}}^{2}}{n_{\mathrm{PBH}}}=f_{\mathrm{PBH}}^{2}\left[\int \frac{d n_{\mathrm{PBH}}}{d M_{\mathrm{PBH}}} d M_{\mathrm{PBH}}\right]^{-1},
\end{aligned}
$$


where we assume that the $\mathrm{PBH}$ abundances are the same for both the MMD and EMD cases. We finally have

$$
M_{\mathrm{eq}}=\left[\int \frac{1}{M_{\mathrm{PBH}}} \frac{d \Phi}{d M_{\mathrm{PBH}}} d M_{\mathrm{PBH}}\right]^{-1} .
$$

We consider two popular EMDs: Lognormal and Powerlaw. The Lognormal EMD [59] is defined by

$$
\frac{d \Phi_{\mathrm{PBH}}}{d M_{\mathrm{PBH}}}=\frac{\exp \left\{-\frac{\ln ^{2}\left(M_{\mathrm{PBH}} / \mu\right)}{2 \sigma^{2}}\right\}}{\sqrt{2 \pi} \sigma M_{\mathrm{PBH}}},
$$

where $\sigma$ and $\mu$ are the standard deviation and mean of the PBH mass, respectively. Such function describes, e.g., the scenario of PBHs forming from a smooth symmetric peak in the inflationary power spectrum $[60,61]$.

The Powerlaw EMD, corresponding to PBHs formed from collapsing cosmic strings or scale-invariant density fluctuations [62], is given by

$$
\frac{d \Phi_{\mathrm{PBH}}}{d M_{\mathrm{PBH}}}=\frac{\mathcal{N}_{\mathrm{PL}}}{M_{\mathrm{PBH}}^{1-\tilde{\gamma}}} \Theta\left(M_{\mathrm{PBH}}-M_{\mathrm{min}}\right) \Theta\left(M_{\mathrm{max}}-M_{\mathrm{PBH}}\right),
$$

characterized by an exponent $\tilde{\gamma} \in(-1,+1)$, a mass interval $\left(M_{\min }, M_{\max }\right)$, and a normalization factor $\mathcal{N}_{\mathrm{PL}} ; \Theta$ is the Heaviside step function.

Dataset and methods.-To extract limits on the PBH abundance from the Lyman- $\alpha$ forest, we adapted the method proposed in Ref. [63]. We built a new grid of hydrodynamic simulations in terms of the properties of PBHs, corresponding to initial linear power spectra featuring a small-scale plateau. Besides that, our analyses rely on a precomputed multidimensional grid of hydrodynamic simulations, associated with several values of the astrophysical and cosmological parameters affecting the Lyman$\alpha$ flux power spectrum. Simulations have been performed with GADGET-III, a modified version of the public code GADGET-II [64,65]. Initial conditions have been produced with 2LPTic [66], at $z=199$, with input linear power spectra for the $\Lambda \mathrm{PBH}$ models obtained by turning on the isocurvature mode in CLASS [67].

Our reference model simulation $[63,68]$ has a box length of $20 / h$ comoving megaparsec with $2 \times 768^{3}$ gas and $\mathrm{CDM}$ particles in a flat $\Lambda$ CDM universe with cosmological parameters as in Ref. [69].

For the cosmological parameters to be varied, we sample different values of $\sigma_{8}$, i.e., the normalization of the linear power spectrum, and $n_{\text {eff }}$, the slope of the power spectrum evaluated at the scale probed by the Lyman- $\alpha$ forest $\left(k_{\alpha}=0.009 \mathrm{~s} / \mathrm{km}\right)$ [70-72]. We included five different simulations for both $\sigma_{8}([0.754,0.904])$ and $n_{\text {eff }}$ ([-2.3474, -2.2674]). Additionally, we included simulations corresponding to different values for the instantaneous reionization redshift, i.e., $z_{\text {reio }}=\{7,9,15\}$.
Regarding the astrophysical parameters, we modeled the IGM thermal history with amplitude $T_{0}$ and slope $\gamma$ of its temperature-density relation, parametrized as $T=$ $T_{0}\left(1+\delta_{\mathrm{IGM}}\right)^{\gamma-1}$, with $\delta_{\mathrm{IGM}}$ being the IGM overdensity [73]. We use simulations with temperatures at mean density $T_{0}(z=4.2)=\{6000,9200,12600\} \mathrm{K}$, evolving with redshift, and a set of three values for the slope of the temperature-density relation, $\gamma(z=4.2)=\{0.88,1.24,1.47\}$. The redshift evolution of both $T_{0}$ and $\gamma$ are parametrized as power laws, such that $T_{0}(z)=T_{0}^{A}\left[(1+z) /\left(1+z_{p}\right)\right]^{T_{0}^{S}}$ and $\gamma(z)=\gamma^{A}\left[(1+z) /\left(1+z_{p}\right)\right]^{\gamma^{S}}$, where the pivot redshift $z_{p}$ is the redshift at which most of the Lyman- $\alpha$ forest pixels are coming from $\left(z_{p}=4.5\right)$. The reference thermal history is defined by $T_{0}(z=4.2)=9200$ and $\gamma(z=4.2)=1.47$ [74].

Furthermore, we considered the effect of ultraviolet (UV) fluctuations of the ionizing background, controlled by the parameter $f_{\mathrm{UV}}$. Its template is built from three simulations with $f_{\mathrm{UV}}=\{0,0.5,1\}$, where $f_{\mathrm{UV}}=0$ corresponds to a spatially uniform UV background [68]. We also included nine grid points obtained by rescaling the mean Lyman- $\alpha$ flux $\bar{F}(z)$-namely, $\{0.6,0.7,0.8,0.9,1.0,1.1$, $1.2,1.3,1.4\} \times \bar{F}_{\text {ref }}$-with reference values given by SDSSIII-BOSS measurements [75]. We also considered eight additional values, obtained by rescaling the optical depth $\tau=-\ln \bar{F}$, i.e., $\{0.6,0.7,0.8,0.9,1.1,1.2,1.3,1.4\} \times \tau_{\text {ref }}$.

Concerning the $\mathrm{PBH}$ properties, we extracted the flux power spectra from 12 hydrodynamic simulations $\left(512^{3}\right.$ particles; 20 comoving $\mathrm{Mpc} / h$ box length) corresponding to the following $\mathrm{PBH}$ mass and fraction products: $\log \left(M_{\mathrm{PBH}} f_{\mathrm{PBH}}\right)=\{1.0,1.5,2.0,2.2,2.3,2.4,2.5,2.6,2.7$, $3.0,3.5,4.0\}$. For this set of simulations, astrophysical and cosmological parameters have been fixed to their reference values, and the equivalent $\Lambda \mathrm{CDM}$ flux power was also determined.

We use an advanced interpolation method, the ordinary kriging method [76], particularly suitable to dealing with the sparse, nonregular grid defined by our simulations. Such a method basically consists of predicting the value of the flux power at a given point by computing a weighted average of all of its known values, with weights inversely proportional to the distance from the considered point. The interpolation is in terms of ratios between the flux power spectra of the $\Lambda \mathrm{PBH}$ models and the reference $\Lambda \mathrm{CDM}$ one. We first interpolate in the astrophysical and cosmological parameter space for the $\Lambda \mathrm{CDM}$ case, then correct all of the $\left(M_{\mathrm{PBH}} f_{\mathrm{PBH}}\right)$ grid points accordingly, and finally interpolate in the $\left(M_{\mathrm{PBH}} f_{\mathrm{PBH}}\right)$ space.

Our datasets are the MIKE and HIRES-KECK samples of quasar spectra, at $z=\{4.2,4.6,5.0,5.4\}$, in ten $k$ bins in the range [0.001-0.08] s/ km, with spectral resolution of 13.6 and $6.7 \mathrm{~s} / \mathrm{km}$ [53]. We consider only measurements at $k>0.005 \mathrm{~s} / \mathrm{km}$ to avoid systematic uncertainties due to continuum fitting. Moreover, we did not use the MIKE 
highest redshift bin [53]. We thus have a total of $49(k, z)$ data points.

Results and discussion. - We obtain our results by maximizing a Gaussian likelihood with a Monte Carlo Markov chain (MCMC) approach, using the publicly available MCMC sampler emcee [77]. We adopted Gaussian priors on the mean fluxes $\bar{F}(z)$, centered on their reference values, with standard deviation $\sigma=0.04$ [68], and on $\sigma_{8}$ and $n_{\text {eff }}$, centered on their Planck values [69], with $\sigma=0.05$, since the final two parameters, whereas well constrained by CMB data, are poorly constrained by Lyman- $\alpha$ data alone [63]. We adopt logarithmic priors on $f_{\mathrm{PBH}} M_{\mathrm{PBH}}$ (but our results are not affected by this choice). Concerning the IGM thermal history, we adopt flat priors on both $T_{0}^{A}$ and $T_{0}^{S}$, in the ranges $[0,2] \times 10^{4} \mathrm{~K}$ and $[-5,5]$, respectively. When the corresponding $T_{0}(z)$ are determined, they can assume values not enclosed by our template of simulations. When this occurs, the corresponding values of the flux power spectra are linearly extrapolated. Regarding $\gamma^{S}$ and $\gamma^{A}$, we impose flat priors on the corresponding $\gamma(z)$ (in the interval $[1,1.7])$. The priors on $z_{\text {reio }}$ and $f_{\mathrm{UV}}$ are flat within the boundaries defined by our grid of simulations.

The limit on the PBH abundance under the MMD assumption corresponds to

$$
f_{\mathrm{PBH}} M_{\mathrm{PBH}} \lesssim 170 M_{\odot}(2 \sigma) .
$$

However, both Planck data and [78] favor $z_{\text {reio }} \sim 8.5$, so we repeated our analysis with a Gaussian prior centered around $z_{\text {reio }}=8.5$, with $\sigma=1.0$; in this case, we have

$$
f_{\mathrm{PBH}} M_{\mathrm{PBH}} \lesssim 60 M_{\odot}(2 \sigma) .
$$

Where all $\mathrm{DM}$ is made by $\mathrm{PBHs}\left(f_{\mathrm{PBH}}=1\right)$, these constraints can be interpreted as absolute limits on the PBH mass. On the other hand, such bounds weaken linearly for smaller $\mathrm{PBH}$ abundances $\left(0.05<f_{\mathrm{PBH}}<1\right)$. The lower limit on $f_{\mathrm{PBH}}$ is given by the fact that, for the monochromatic case, at $z=199$, i.e., the redshift of the initial conditions of our simulations, if $f_{\mathrm{PBH}}$ is smaller, the Poisson effect is subdominant with respect to the so-called seed effect, the treatment of which goes beyond our purposes.

The degeneracy between $z_{\text {reio }}$ and the PBH mass can be understood as follows: a higher reionization redshift corresponds to a more effective filtering scale, and thus to a power suppression compensated for by larger values of the PBH mass.

In Fig. 2, we show the $1 \sigma$ and $2 \sigma$ contours for some of the parameters of our analyses, for both prior choices on $z_{\text {reio }}$. The degeneracy between the amplitude of the IGM temperature $T_{0}^{A}\left(z=z_{p}\right)$ and the $\mathrm{PBH}$ mass derives from the opposite effects on the flux power spectra due to the increase of the two parameters. A hotter IGM implies a small-scale power suppression which is balanced by increasing $M_{\mathrm{PBH}} f_{\mathrm{PBH}}$. Slightly larger values for the mean

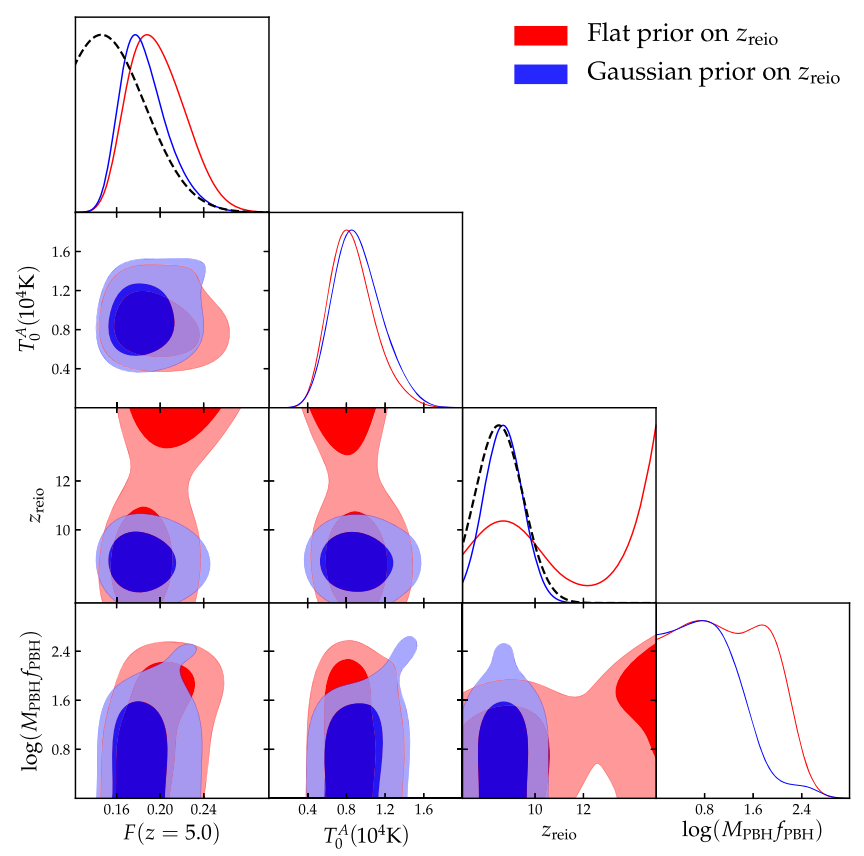

FIG. 2. $1 \sigma$ and $2 \sigma$ contour plots for some of the parameters of our analyses, for the two different prior choices on $z_{\text {reio }}$. The values for $M_{\mathrm{PBH}}$ are expressed in units of $M_{\odot}$. The dashed lines correspond to the Gaussian priors that we used for some of our analyses.

fluxes $\bar{F}(z)$ are also required for accommodating the power enhancement induced by relatively large values of the PBH mass. The dashed lines represent the Gaussian priors imposed on $\bar{F}(z=5)$ and $z_{\text {reio }}$, with the latter referring to the blue plots. Note that our MCMC analyses favor higher values for $\bar{F}(z=5)$ (still in agreement with its prior distribution), allowing in turn a larger power enhancement due to PBHs.

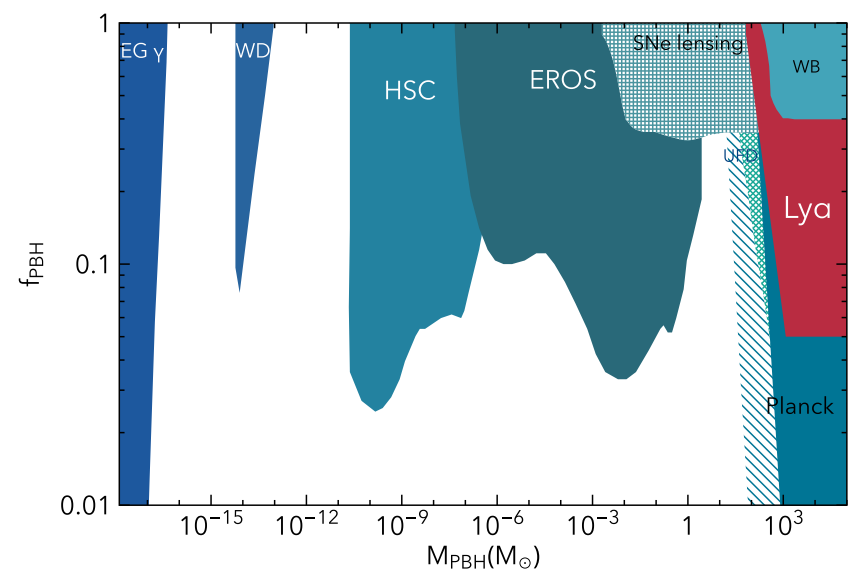

FIG. 3. Present experimental constraints on the PBH abundance for MMDs (from Refs. [31,42,82,83]) are in shades of blue, while in red are limits from this Letter. Patterned areas show limits that are the most dependent on astrophysical assumptions [79]. 

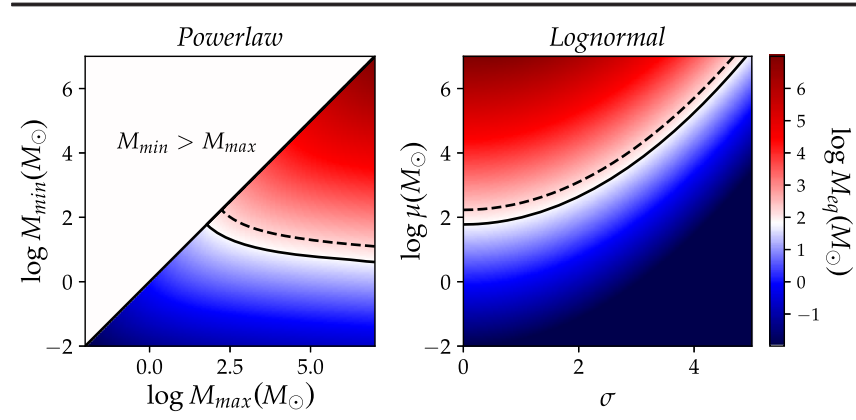

we ruled out a large part of the parameter space for two of the most popular EMDs: Powerlaw and Lognormal.

In the near future, it is expected that a larger number of high-redshift, high-resolution, and signal-to-noise quasar spectra collected with the ESPRESSO spectrograph [85] or at the E-ELT could allow one to achieve tighter constraints.

Whereas PBHs with mass $O(10)$ can potentially solve some tensions in the cosmic infrared background [86-88], the accumulation of limits on the PBHs as a DM model in the mass range probed by LIGO seems to suggest that the hypothesis of $30 M_{\odot}$ PBHs being the DM is less and less likely to be true.

It has, however, become clear that these studies brought a plethora of astrophysical information, and even the exclusion of certain PBH mass ranges will bring information on some of the processes happening in the very early Universe.

In Fig. 3, we report the updated plot with the constraints on the DM fraction in PBHs, in the monochromatic case. The "LIGO window" between $\sim 20 M_{\odot}$ and $80 M_{\odot}$ initially suggested in Ref. [15] has been probed and tentatively closed by constraints from ultrafaint dwarf galaxies [35] and supernovae lensing [31]; these constraints have been questioned because of astrophysics uncertainties (see, e.g., Refs. [79-81]): we show them in a patterned area. In this Letter, we robustly close the higher mass part of that remaining window. There remains, however, an interesting possibility in the very low mass range, $\lesssim 10^{-10} M_{\odot}[82,84]$.

By defining an equivalent mass $M_{\text {eq }}$, one can convert the limits for the MMD case to bounds on the parameters of a given EMD. In Fig. 4, we provide such bounds, as was shown in Fig. 3 of Ref. [58] for other observational constraints. The left panel shows the Powerlaw EMD, with $\tilde{\gamma}=0$, focusing on the following mass range: $M_{\min }$, $M_{\max } \in\left[10^{-2}, 10^{7}\right] M_{\odot}$. In the right panel, we show the Lognormal EMD, scanning the parameter space defined by $\mu \in\left[10^{-2}, 10^{7}\right] M_{\odot}$ and $\sigma \in[0,5]$. The two black lines correspond to the contraints quoted above, i.e., $M_{\text {eq }}=$ $60 M_{\odot}$ (solid lines), and $M_{\text {eq }}=170 M_{\odot}$ (dashed lines). The blue regions are admitted by our analyses, while the red areas are ruled out.

Conclusions. - In this Letter, we have presented new bounds on the DM fraction in PBHs, using an extensive analysis of high-redshift Lyman- $\alpha$ forest data, improving over previous similar analyses in three different ways: (1) We used the high-resolution MIKE and HIRES data, exploring better the high-redshift range where primordial differences are more prominent. (2) We relied on very accurate high-resolution hydrodynamic simulations which expand over a thermal history suggested by data. (3) We used the full shape of the 1D flux power rather than a single amplitude parameter.

Our results improve previous constraints by roughly 2 orders of magnitude; furthermore, we have generalized our results to nonmonochromatic $\mathrm{PBH}$ mass distributions, and

The authors are thankful to Nils Schöneberg, Nicola Bellomo, José Luis Bernal, Pasquale D. Serpico, Takeshi Kobayashi, Emiliano Sefusatti, and Gabrijela Zaharijas for the helpful discussions. R. M., G.S, and M. V. are supported by the INFN INDARK PD51 Grant. The simulations were performed on the Ulysses SISSA/ICTP supercomputer.

[1] S. Hawking, Mon. Not. R. Astron. Soc. 152, 75 (1971).

[2] P. Ivanov, P. Naselsky, and I. Novikov, Phys. Rev. D 50, 7173 (1994).

[3] J. García-Bellido, A. Linde, and D. Wands, Phys. Rev. D 54, 6040 (1996).

[4] P. Ivanov, Phys. Rev. D 57, 7145 (1998).

[5] A. Polnarev and R. Zembowicz, Phys. Rev. D 43, 1106 (1991).

[6] S. Hawking, Phys. Lett. B 231, 237 (1989).

[7] U. F. Wichoski, J. H. MacGibbon, and R. H. Brandenberger, Phys. Rep. 307, 191 (1998).

[8] V. Berezin, V. Kuzmin, and I. Tkachev, Phys. Lett. B 120B, 91 (1983).

[9] J. Ipser and P. Sikivie, Phys. Rev. D 30, 712 (1984).

[10] M. Crawford and D. N. Schramm, Nature (London) 298, 538 (1982).

[11] D. La and P. J. Steinhardt, Phys. Lett. B 220, 375 (1989).

[12] S. Shandera, D. Jeong, and H. S. Grasshorn Gebhardt, Phys. Rev. Lett. 120, 241102 (2018).

[13] B. P. Abbott et al. (LIGO Scientific and Virgo Collaborations), Phys. Rev. Lett. 116, 061102 (2016).

[14] B. P. Abbott et al. (LIGO Scientific and Virgo Collaborations), Phys. Rev. Lett. 116, 241102 (2016).

[15] S. Bird, I. Cholis, J. B. Muñoz, Y. Ali-Haïmoud, M. Kamionkowski, E. D. Kovetz, A. Raccanelli, and A. G. Riess, Phys. Rev. Lett. 116, 201301 (2016).

[16] A. Raccanelli, E. D. Kovetz, S. Bird, I. Cholis, and J. B. Muñoz, Phys. Rev. D 94, 023516 (2016).

[17] G. Scelfo, N. Bellomo, A. Raccanelli, S. Matarrese, and L. Verde, J. Cosmol. Astropart. Phys. 09 (2018) 039. 
[18] I. Cholis, E. D. Kovetz, Y. Ali-Haïmoud, S. Bird, M. Kamionkowski, J. B. Muñoz, and A. Raccanelli, Phys. Rev. D 94, 084013 (2016).

[19] E. D. Kovetz, I. Cholis, P. C. Breysse, and M. Kamionkowski, Phys. Rev. D 95, 103010 (2017).

[20] E. D. Kovetz, Phys. Rev. Lett. 119, 131301 (2017).

[21] J. B. Muñoz, E. D. Kovetz, L. Dai, and M. Kamionkowski, Phys. Rev. Lett. 117, 091301 (2016).

[22] A. Barnacka, J.-F. Glicenstein, and R. Moderski, Phys. Rev. D 86, 043001 (2012).

[23] A. Katz, J. Kopp, S. Sibiryakov, and W. Xue, J. Cosmol. Astropart. Phys. 12 (2018) 005.

[24] K. Griest, A. M. Cieplak, and M. J. Lehner, Astrophys. J. 786, 158 (2014).

[25] H. Niikura, M. Takada, N. Yasuda, R. H. Lupton, T. Sumi, S. More, A. More, M. Oguri, and M. Chiba, Nat. Astron. 3, 524 (2019).

[26] P. Tisserand et al. (EROS-2 Collaboration), Astron. Astrophys. 469, 387 (2007).

[27] S. Calchi Novati, S. Mirzoyan, P. Jetzer, and G. Scarpetta, Mon. Not. R. Astron. Soc. 435, 1582 (2013).

[28] C. Alcock et al. (MACHO Collaboration), Astrophys. J. Lett. 550, L169 (2001).

[29] E. Mediavilla, J. A. Munoz, E. Falco, V. Motta, E. Guerras, H. Canovas, C. Jean, A. Oscoz, and A. M. Mosquera, Astrophys. J. 706, 1451 (2009).

[30] P. N. Wilkinson, D. R. Henstock, I. W. A. Browne, A. G. Polatidis, P. Augusto, A. C. S. Readhead, T. J. Pearson, W. $\mathrm{Xu}, \mathrm{G}$. B. Taylor, and R. C. Vermeulen, Phys. Rev. Lett. 86, 584 (2001).

[31] M. Zumalacarregui and U. Seljak, Phys. Rev. Lett. 121, 141101 (2018).

[32] P. W. Graham, S. Rajendran, and J. Varela, Phys. Rev. D 92 , 063007 (2015).

[33] F. Capela, M. Pshirkov, and P. Tinyakov, Phys. Rev. D 87, 123524 (2013).

[34] D. P. Quinn, M. I. Wilkinson, M. J. Irwin, J. Marshall, A. Koch, and V. Belokurov, Mon. Not. R. Astron. Soc. Lett. 396, L11 (2009).

[35] T. D. Brandt, Astrophys. J. Lett. 824, L31 (2016).

[36] M. Raidal, V. Vaskonen, and H. Veermäe, J. Cosmol. Astropart. Phys. 09 (2017) 037.

[37] Y. Ali-Haïmoud, E. D. Kovetz, and M. Kamionkowski, Phys. Rev. D 96, 123523 (2017).

[38] M. Raidal, C. Spethmann, V. Vaskonen, and H. Veermäe, J. Cosmol. Astropart. Phys. 02 (2019) 018.

[39] R. Magee, A.-S. Deutsch, P. McClincy, C. Hanna, C. Horst, D. Meacher, C. Messick, S. Shandera, and M. Wade, Phys. Rev. D 98, 103024 (2018).

[40] D. Gaggero, G. Bertone, F. Calore, R. M. T. Connors, M. Lovell, S. Markoff, and E. Storm, Phys. Rev. Lett. 118, 241101 (2017).

[41] M. Ricotti, J. P. Ostriker, and K. J. Mack, Astrophys. J. 680, 829 (2008).

[42] Y. Ali-Haïmoud and M. Kamionkowski, Phys. Rev. D 95, 043534 (2017).

[43] V. Poulin, P. D. Serpico, F. Calore, S. Clesse, and K. Kohri, Phys. Rev. D 96, 083524 (2017).

[44] J. L. Bernal, N. Bellomo, A. Raccanelli, and L. Verde, J. Cosmol. Astropart. Phys. 10 (2017) 052.
[45] D. Aloni, K. Blum, and R. Flauger, J. Cosmol. Astropart. Phys. 05 (2017) 017.

[46] N. Bellomo, J. L. Bernal, A. Raccanelli, and L. Verde, J. Cosmol. Astropart. Phys. 01 (2018) 004.

[47] T. Nakama, J. Silk, and M. Kamionkowski, Phys. Rev. D 95, 043511 (2017).

[48] M. Sasaki, T. Suyama, T. Tanaka, and S. Yokoyama, Classical Quantum Gravity 35, 063001 (2018).

[49] B. Carr and J. Silk, Mon. Not. R. Astron. Soc. 478, 3756 (2018).

[50] Y. Ali-Haimoud et al., arXiv:1903.04424.

[51] M. Viel, S. Matarrese, H. J. Mo, M. G. Haehnelt, and T. Theuns, Mon. Not. R. Astron. Soc. 329, 848 (2002).

[52] M. Viel, J. Lesgourgues, M. G. Haehnelt, S. Matarrese, and A. Riotto, Phys. Rev. D 71, 063534 (2005).

[53] M. Viel, G. D. Becker, J. S. Bolton, and M. G. Haehnelt, Phys. Rev. D 88, 043502 (2013).

[54] N. Afshordi, P. McDonald, and D. N. Spergel, Astrophys. J. 594, L71 (2003).

[55] P. Meszaros, Astron. Astrophys. 38, 5 (1975).

[56] J.-O. Gong and N. Kitajima, J. Cosmol. Astropart. Phys. 08 (2017) 017.

[57] A. Kalaja et al. (to be published).

[58] B. Carr, M. Raidal, T. Tenkanen, V. Vaskonen, and H. Veermae, Phys. Rev. D 96, 023514 (2017).

[59] A. Dolgov and J. Silk, Phys. Rev. D 47, 4244 (1993).

[60] A. M. Green, Phys. Rev. D 94, 063530 (2016).

[61] K. Kannike, L. Marzola, M. Raidal, and H. Veermäe, J. Cosmol. Astropart. Phys. 09 (2017) 020.

[62] B. J. Carr, Astrophys. J. 201, 1 (1975).

[63] R. Murgia, V. Iršič, and M. Viel, Phys. Rev. D 98, 083540 (2018).

[64] V. Springel, N. Yoshida, and S. D. M. White, New Astron. 6, 79 (2001).

[65] V. Springel, Mon. Not. R. Astron. Soc. 364, 1105 (2005).

[66] M. Crocce, S. Pueblas, and R. Scoccimarro, Mon. Not. R. Astron. Soc. 373, 369 (2006).

[67] D. Blas, J. Lesgourgues, and T. Tram, J. Cosmol. Astropart. Phys. 07 (2011) 034.

[68] V. Iršič et al., Phys. Rev. D 96, 023522 (2017).

[69] P. A. R. Ade et al. (Planck observations Collaboration), Astron. Astrophys. 594, A13 (2016).

[70] U. Seljak, A. Slosar, and P. McDonald, J. Cosmol. Astropart. Phys. 10 (2006) 014.

[71] P. McDonald et al. (SDSS Collaboration), Astrophys. J. Suppl. Ser. 163, 80 (2006).

[72] A. Arinyo-i Prats, J. Miralda-Escude, M. Viel, and R. Cen, J. Cosmol. Astropart. Phys. 12 (2015) 017.

[73] L. Hui and N. Y. Gnedin, Mon. Not. R. Astron. Soc. 292, 27 (1997).

[74] J. S. Bolton, E. Puchwein, D. Sijacki, M. G. Haehnelt, T.-S. Kim, A. Meiksin, J. A. Regan, and M. Viel, Mon. Not. R. Astron. Soc. 464, 897 (2017).

[75] N. Palanque-Delabrouille et al., Astron. Astropart. 559, A85 (2013).

[76] R. Webster and M. A. Oliver, Geostatistics for Environmental Scientists (John Wiley \& Sons, New York, 2007).

[77] D. Foreman-Mackey, D. W. Hogg, D. Lang, and J. Goodman, Publ. Astron. Soc. Pac. 125, 306 (2013). 
[78] E. Boera, G. D. Becker, J. S. Bolton, and F. Nasir, Astrophys. J. 872, 101 (2019).

[79] B. Carr, F. Kühnel, and M. Sandstad, Phys. Rev. D 94, 083504 (2016).

[80] T. S. Li et al. (DES Collaboration), Astrophys. J. 838, 8 (2017).

[81] See also the Primordial versus Astrophysical Origin of Black Holes CERN workshop.

[82] N. Bartolo, V. De Luca, G. Franciolini, M. Peloso, D. Racco, and A. Riotto, Phys. Rev. D 99, 103521 (2019).
[83] B. J. Carr, K. Kohri, Y. Sendouda, and J. Yokoyama, Phys. Rev. D 81, 104019 (2010).

[84] S. Pi, Y.-1. Zhang, Q.-G. Huang, and M. Sasaki, J. Cosmol. Astropart. Phys. 05 (2018) 042.

[85] F. Pepe et al., Messenger 153, 6 (2013).

[86] A. Kashlinsky, Astrophys. J. 823, L25 (2016).

[87] A. Kashlinsky, Phys. Rep. 409, 361 (2005).

[88] A. Kashlinsky, R. G. Arendt, J. C. Mather, and S. H. Moseley, Nature (London) 438, 45 (2005). 